\title{
Perfil de pacientes idosos e tempo de permanência em ambulatório geronto-geriátrico
}

\section{Profileof agedatpetientsand duration of stay at agerontologicgeriatricdinic}

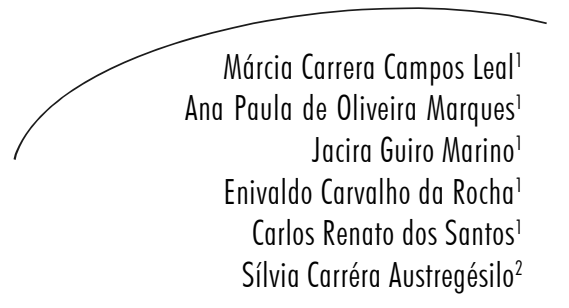

\section{Resumo}

Objetivo: Identificar o perfil de pacientes idosos em assistência ambulatorial no Núcleo de Atenção ao Idoso e as variáveis que interferem no tempo de permanência no serviço. Métodos: Realizou-se pesquisa quantitativa, descritiva, de corte transversal, baseada na análise de 1.194 prontuários médicos de pacientes atendidos no período de 2000 a 2004. As variáveis foram: dados sociodemográficos, registros da avaliação médica e tempo de permanência do idoso no ambulatório. Para análise da relação entre variáveis clínicas e tempo de permanência, utilizou-se o modelo de regressão logística. Resultados: Em todas as faixas etárias, as mulheres (76,5\%) foram mais numerosas que os homens (23,5\%). Quanto à escolaridade, $12,9 \%$ eram iletrados e a categoria mais prevalente tinha primeiro grau incompleto $(37,1 \%)$. O número de idosas viúvas $(38 \%)$ foi três vezes maior que o de viúvos $(12 \%)$. A maioria dos idosos $(94,4 \%)$ tinha companhia de moradia e $84,4 \%$ não tinham plano de saúde. A média do tempo de permanência do idoso no serviço foi 2 anos e 8 meses, com desvio padrão de 1,5 ano. As variáveis que mais influenciaram o tempo de permanência foram: idade, queixa de tontura, distúrbios refracionais, auditivos, urinários, catarata, fraturas ósseas e antecedentes cirúrgicos. Conclusões: Por regressão logística, identificou-se que antecedentes cirúrgicos, distúrbios auditivos, urinários e fraturas ósseas atuaram aumentando o tempo de permanência, mas queixa de tontura, distúrbios refracionais e catarata reduziram a permanência do idoso no serviço.

Universidade Federal de Pernambuco, Centro de Ciências da Saúde. Recife, PE, Brasil

1 Departamento de Medicina Social

2 Departamento de Prótese e Cirurgia Bucofacial

Correspondência / Correspondence

Márcia Carréra Campos Leal

UFPE - Departamento de Medicina Social

Av. Prof. Moraes Rêgo, $s / n^{\circ}$

50670-901, Recife, PE, Brasil

E-mail: marciacarrera@hotmail.com
Palavras-chave: Idoso. Perfil de Saúde. Assistência Ambulatorial. Assistência de Longa Duração. Epidemiologia Descritiva. Modelos Logísticos. Estudos Transversais. Serviço de Saúde. Morbidade. Tempo de Permanência. 
Abstract

Objective: To identify the profile of aged outpatients attended at the Núcleo de Atenção ao Idoso and the variables that interfere on duration of stay in the service. Methods: A quantitative, descriptive, cross-sectional research was carried through, based on the analysis of 1,194 medical reports of aged outpatients attended from 2000 up to 2004. The variables were: sociodemographic data, clinical registers in medical evaluation and duration of stay of the aged outpatients at the clinic. A logistic regression was performed to analyze the relation between clinical variables and time of stay. Results: Women $(76.5 \%)$ were more numerous than men $(23.5 \%)$, in all age intervals. According to scholarship, $12.9 \%$ were illiterate and the most prevalent category $(37.1 \%)$ had incomplete first degree. The number of aged widower women $(38 \%)$ was three times those of widower men $(12 \%)$. The majority of aged $(94.4 \%)$ had a company of housing and $84.4 \%$ did not have health plan.

Key words: Aged. Health Profile. Ambulatory Care. Long-Term Care. Epidemiology, Descriptive. Logistic Models. CrossSectional Studies. Service of Health. Morbidity. Duration of Stay.
INTRODUÇÃO

No Brasil, a população idosa, considerada pela Política Nacional do Idoso como aquela constituída por indivíduos com 60 anos e mais, compõe hoje o segmento populacional que mais cresce em termos proporcionais. ${ }^{1} \mathrm{De}$ acordo com as projeções estatísticas, até o ano de 2025, o Brasil ocupará a sexta posição mundial em termos de população idosa, com mais de 32 milhões de indivíduos nesta faixa etária, representando $15 \%$ da população total. ${ }^{2,3}$

A passagem de uma situação de alta fecundidade e alta mortalidade para uma de baixa fecundidade e progressiva diminuição da mortalidade favoreceu o envelhecimen- to da população brasileira, com implicações no panorama epidemiológico. ${ }^{4,5}$ Nesse novo cenário, as doenças transmissíveis tendem a diminuir sua incidência, enquanto as doenças crônicas não-transmissíveis apresentam prevalência elevada, sobretudo entre os mais velhos. ${ }^{6,7}$

As alterações observadas no perfil de morbidade e mortalidade decorrentes do envelhecimento geram demandas específicas por cuidados de saúde dirigidos ao segmento idoso. Este utiliza os serviços de saúde de forma mais intensiva quando comparado a outros grupos etários, em função da maior ocorrência de enfermidades crônicas e possível perda funcional com o avanço dos anos. $5,6,8,9$ 
De acordo com Ramos, ${ }^{9}$ o grande desafio deste século será cuidar de uma população envelhecida, em sua maioria com nível socioeconômico e educacional baixo e alta prevalência de doenças incapacitantes.

Em relação aos cuidados com o segmento mais envelhecido, vale destacar a urgência em termos de reestruturação dos espaços sociais no acolhimento adequado à pessoa idosa, incluindo os serviços de saúde e a formação de profissionais devidamente qualificados, para promoção, proteção e recuperação da saúde daqueles que, superando as adversidades ao longo da vida, conseguiram envelhecer.

Nesta perspectiva, a Universidade Federal de Pernambuco implantou o Núcleo de Atenção ao Idoso (NAI), unidade ambulatorial que presta atendimento geronto-geriátrico a indivíduos com idade equivalente ou superior a 60 anos, tendo por premissa a melhoria da qualidade de vida dos idosos assistidos, mediante atendimento individual ou em grupo, com orientação a familiares e cuidadores.

O presente estudo tem por objetivo identificar o perfil do idoso que demanda o serviço e estimar seu tempo de permanência no mesmo.

\section{MÉTODOS}

O estudo foi desenvolvido no Núcleo de Atenção ao Idoso (NAI), localizado no Centro de Ciências da Saúde da Universi- dade Federal de Pernambuco (UFPE), vinculado administrativamente ao Programa do Idoso (PROIDOSO) e ambos, a Pró-Reitoria de Extensão (PROEXT).

O NAI caracteriza-se como uma unidade de saúde de natureza ambulatorial, que presta atendimento em equipe multidisciplinar a pessoas idosas (60 anos e mais), mediante consulta individual e em grupo, esta última realizada por meio de oficinas temáticas, além de contribuir com a formação discente e capacitação de recursos humanos na atenção geronto-geriátrica.

De natureza descritiva e corte transversal, o estudo foi realizado a partir de dados secundários relativos aos prontuários existentes no serviço, desde sua criação em 2000 até 2004. O enfoque metodológico consistiu na análise de 1.194 prontuários médicos. Para tal, foi desenvolvida uma plataforma em Access, possibilitando a atualização e o acompanhamento sistemático das informações, posterior à implantação do banco de dados. ${ }^{10}$

As informações de interesse para o estudo incluíram dados sociodemográficos e os registros clínicos da avaliação médica mais prevalentes, obtidos dos prontuários existentes no serviço. As variáveis sociodemográficas corresponderam a: idade (em anos completos, agrupados por faixa etária), sexo, escolaridade (distribuída em sete categorias: não-analfabetizado, alfabetizado, $1^{\circ} \mathrm{grau}$ incompleto, $1^{\circ}$ grau completo, $2^{\circ}$ grau incompleto, $2^{\circ}$ grau completo e $3^{\circ}$ grau), convivência no domicílio (mora acompanhado 
ou sozinho), estado civil (casado/companheiro, separado/divorciado, solteiro, viúvo) e condição de ter plano de saúde.

Em virtude da existência de dados perdidos (missing values) para algumas variáveis, houve pequena variação no número de informações processadas em determinadas categorias.

Para estimar o tempo de permanência do idoso no serviço, foi feita inicialmente uma análise individual da referida variável, calculando-se o tempo médio de permanência e seu desvio padrão. Em virtude do elevado coeficiente de variação apresentado e da assimetria observada no histograma, optou-se pela análise de dados aparados, ${ }^{2}$ que consiste em extrair um pequeno percentual de dados nos extremos da amostra. Desta forma, retiraram-se $2,5 \%$ dos maiores e $2,5 \%$ dos menores valores da variável, sendo recalculados a média, o desvio padrão e o histograma.
Considerando que os dados sociodemográficos e os registros clínicos mais prevalentes obtidos dos prontuários (variáveis independentes) podem influenciar o tempo de permanência do idoso no serviço (variável dependente), o modelo foi ajustado mediante análise de regressão logística, sendo o tempo de permanência transformado em variável dicotômica, a saber: permanência no ambulatório abaixo de um ano e acima de um ano. ${ }^{11}$

\section{RESULTADOS}

No que diz respeito à caracterização dos idosos por sexo e faixa etária (tabela 1), as mulheres são maioria $(76,5 \%)$ em relação aos homens idosos $(23,5 \%)$ e também predominam no conjunto dos prontuários analisados (1.181) nos intervalos etários mais distais ( 80 anos e mais), correspondendo a $13 \%$ da casuística, enquanto que os homens mais idosos equivalem a apenas 5,5\%. 
Tabela 1 - Distribuição de idosos segundo variáveis sociodemográficas. Núcleo de Atenção ao Idoso - NAI/ UFPE. Recife, 2000 - 2004.

\begin{tabular}{|c|c|c|c|c|c|c|c|}
\hline & \multirow{3}{*}{ Variáveis } & \multicolumn{4}{|c|}{ Sexo } & \multirow{2}{*}{\multicolumn{2}{|c|}{ Total }} \\
\hline & & \multicolumn{2}{|c|}{ Homem } & \multicolumn{2}{|c|}{ Mulher } & & \\
\hline \multirow{6}{*}{ Idade (anos) } & & $\%$ & $\mathrm{~N}$ & $\%$ & $\mathrm{~N}$ & $\%$ & $\mathrm{~N}$ \\
\hline & 60 a 69 & 33,5 & 93 & 42,4 & 383 & 40,3 & 476 \\
\hline & 70 a 79 & 43,2 & 120 & 40,6 & 367 & 41,3 & 487 \\
\hline & 80 a 89 & 18,3 & 51 & 13,5 & 122 & 14,6 & 173 \\
\hline & 90 anos e mais & 5,0 & 14 & 3,4 & 31 & 3,8 & 45 \\
\hline & Total & 23,5 & 278 & 76,5 & 903 & & 1181 \\
\hline \multirow{8}{*}{ Escolaridade } & Não alfabetizado & 10,1 & 28 & 13,7 & 124 & 12,9 & 152 \\
\hline & Alfabetizado & 10,5 & 29 & 10,8 & 98 & 10,8 & 127 \\
\hline & $1^{\circ}$ grau incompleto & 37,7 & 104 & 36,9 & 334 & 37,1 & 438 \\
\hline & $1^{\mathrm{o}}$ grau completo & 11,2 & 31 & 11,6 & 105 & 11,5 & 136 \\
\hline & $2^{\circ}$ grau incompleto & 2,9 & 8 & 4,8 & 43 & 4,3 & 51 \\
\hline & $2^{\circ}$ grau completo & 16,7 & 46 & 13,2 & 119 & 14,0 & 165 \\
\hline & $3^{\circ}$ grau & 10,9 & 30 & 9,0 & 81 & 9,4 & 111 \\
\hline & Total & & 276 & & 904 & & 1180 \\
\hline \multirow{3}{*}{$\begin{array}{l}\text { Convivência no } \\
\text { domicílio }\end{array}$} & Mora acompanhado & 96,8 & 270 & 93,7 & 857 & 94,4 & 1127 \\
\hline & Mora sozinho & 3,2 & 9 & 6,3 & 58 & 5,6 & 67 \\
\hline & Total & & 279 & & 915 & & 1194 \\
\hline \multirow{5}{*}{ Estado Civil } & $\begin{array}{l}\text { Casado ou tem } \\
\text { companheiro (a) }\end{array}$ & 76,9 & 213 & 35,0 & 316 & 44,8 & 529 \\
\hline & Separado / divorciado (a) & 5,1 & 14 & 6,8 & 61 & 6,4 & 75 \\
\hline & Solteiro (a) & 6,1 & 17 & 19,4 & 175 & 16,3 & 192 \\
\hline & Viúvo (a) & 11,9 & 33 & 38,9 & 351 & 32,5 & 384 \\
\hline & Total & & 277 & & 903 & & 1180 \\
\hline \multirow{3}{*}{$\begin{array}{l}\text { Tem plano de } \\
\text { saúde }\end{array}$} & Sim & 10,4 & 27 & 17,2 & 149 & 15,6 & 176 \\
\hline & Não & 89,6 & 233 & 82,8 & 719 & 84,4 & 952 \\
\hline & Total & & 260 & & 868 & & 1128 \\
\hline
\end{tabular}

Quanto à escolaridade, verifica-se que $12,9 \%$ dos idosos que buscam atendimento em saúde no NAI são analfabetos. Em relação às outras categorias analisadas, o primeiro grau incompleto aparece como a mais prevalente, correspondendo a $37,1 \%$.

No que diz respeito à condição de convivência no domićlilio, a grande maioria dos idosos $(94,4 \%)$ mora acompanhada.
Em relação ao estado civil, verifica-se que $76,9 \%$ dos homens são casados ou têm companheira, enquanto apenas $35 \%$ das mulheres se enquadram nessa categoria. Situação inversa é observada na condição de viuvez, quando o número de viúvas $(38,5 \%)$ é superior a três vezes o número de homens viúvos (11,9\%).

No tocante à condição de ter plano de saúde, cabe destacar a grande totalidade de 
idosos $(84,4 \%)$ que não se enquadram nessa categoria.

Os registros clínicos mais prevalentes identificados nos prontuários médicos (tabela 2), considerando ambos os sexos, correspondem a: hipertensão arterial
(43\%), problemas de visão (30\%) dislipidemias $(25 \%)$, constipação intestinal $(18,6 \%)$, antecedentes cirúrgicos (16,7\%) queixa de tontura $(16,5 \%)$, catarata $(14,9 \%)$, fraturas ósseas $(14,5 \%)$ problemas urinários $(11,9 \%)$, problemas de audição $(8,2 \%)$.

Tabela 2 - Distribuição de idosos segundo registros clínicos mais frequentes nos prontuários médicos. Núcleo de Atenção ao Idoso - NAI/UFPE. Recife, 2000 -2004.

\begin{tabular}{|c|c|c|c|}
\hline \multicolumn{2}{|c|}{ Condição Clínica Registrada em Prontuário } & \multirow{2}{*}{$\begin{array}{c}\% \\
43,0\end{array}$} & \multirow{2}{*}{$\begin{array}{l}\mathrm{N} \\
514\end{array}$} \\
\hline \multirow{3}{*}{ Hipertensão } & $\operatorname{Sim}$ & & \\
\hline & Não & 57,0 & 680 \\
\hline & Total & 100,0 & 1194 \\
\hline \multirow{3}{*}{ Problemas de visão } & $\operatorname{Sim}$ & 30,0 & 358 \\
\hline & Não & 70,0 & 836 \\
\hline & Total & 100,0 & 1194 \\
\hline \multirow{3}{*}{ Dislipidemias } & $\operatorname{Sim}$ & 25,0 & 298 \\
\hline & Não & 75,0 & 896 \\
\hline & Total & 100,0 & 1194 \\
\hline \multirow{3}{*}{ Constipação Intestinal } & Sim & 18,6 & 222 \\
\hline & Não & 81,4 & 972 \\
\hline & Total & 100,0 & 1194 \\
\hline \multirow{3}{*}{ Antecedentes cirúrgicos } & $\operatorname{Sim}$ & 16,7 & 199 \\
\hline & Não & 83,3 & 995 \\
\hline & Total & 100,0 & 1194 \\
\hline \multirow{3}{*}{ Queixa de tontura } & Sim & 16,5 & 197 \\
\hline & Não & 83,5 & 997 \\
\hline & Total & 100,0 & 1194 \\
\hline \multirow{3}{*}{ Catarata } & Sim & 14,9 & 178 \\
\hline & Não & 85,1 & 1016 \\
\hline & Total & 100,0 & 1194 \\
\hline \multirow{3}{*}{ Fraturas ósseas } & Sim & 14,5 & 173 \\
\hline & Não & 85,5 & 1021 \\
\hline & Total & 100,0 & 1194 \\
\hline \multirow{3}{*}{ Problemas urinários } & Sim & 11,9 & 142 \\
\hline & Não & 88,1 & 1052 \\
\hline & Total & 100,0 & 1194 \\
\hline \multirow{3}{*}{ Problemas de audição } & $\operatorname{Sim}$ & 8,2 & 98 \\
\hline & Não & 91,8 & 1096 \\
\hline & Total & 100,0 & 1194 \\
\hline
\end{tabular}


Quanto ao tempo de permanência da pessoa idosa no ambulatório, a análise individual das variáveis identificou elevado coeficiente de variação, tendo sido retira- dos os valores extremos (5\%), reduzindo assim a variância sem alterar a média, conforme visto nos histogramas dos dois conjuntos de dados (gráfico 1).

Gráfico 1 - Histograma dos anos de permanência no serviço

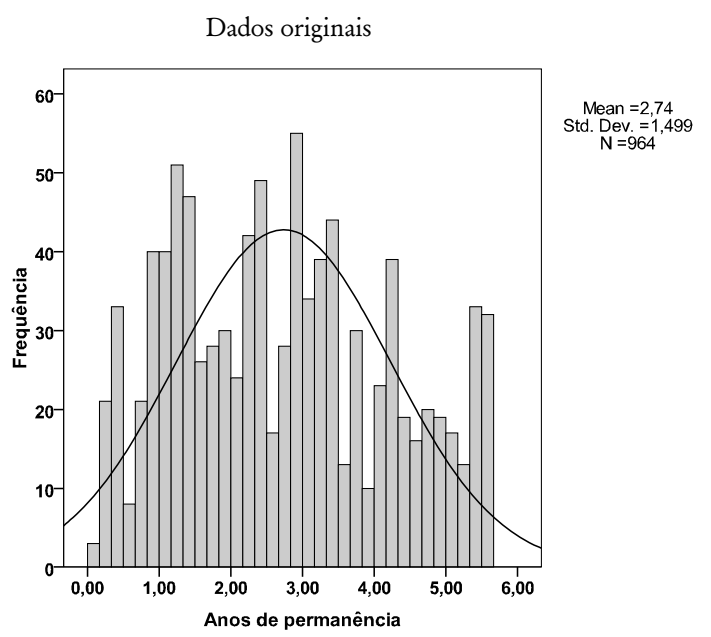

Este procedimento se mostrou adequado, pois a média e o desvio padrão não se alteraram e o histograma se tornou mais simétrico.

As variáveis idade, queixa de tontura, problemas de visão, audição, urinários, catarata, fraturas ósseas e antecedentes cirúrgicos foram identificadas como as mais significativas, apresentando o $\mathrm{R}^{2}$ (Nagelkerke) igual a 0,668. A estatística do teste de bom ajustamento (goodness-of-fit) apresenta o valor 10,492 , distribuída como uma $\chi_{8}^{2}$, com significância de 0,232, implicando o bom ajustamento do modelo. ${ }^{12}$

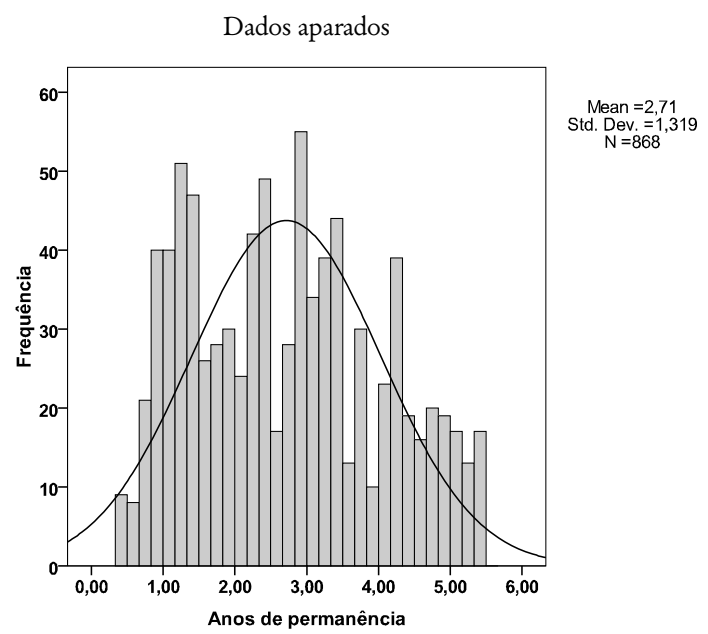

O modelo é dado por:

$\mathrm{y}_{\mathrm{t}}=-(0,94 *$ idade $)-(0,696 *$ tonturas $)-$ $(0,896$ *visão $)+(1,052 *$ audição $)+$ $+(1,193 *$ urinária $)-(2,756 *$ catarata $)+$ $(2,485 *$ fraturas ósseas $)+\left(1,306^{*}\right.$ antecedentes cirúrgicos)

(1)

\section{DISCUSSÃO}

A procura das mulheres por assistência à saúde de forma mais sistemática e contínua ao longo da vida tem sido sugerida como 
um dos fatores explicativos para a maior longevidade feminina e responsável pela predominância das mulheres nos serviços de saúde,,$^{2,4,7}$ fato também observado neste estudo, no qual as mulheres representam a maioria absoluta da casuística investigada.

A frequência mais elevada de mulheres com idade entre 60 a 69 anos (42,4\%), consideradas idosas jovens de acordo com a classificação adotada por Veras, ${ }^{8}$ em relação aos homens $(33,5 \%)$ e nas demais faixas etárias analisadas, também é verificado em outros estudos com amostras provenientes de serviços de saúde ou de base populacional. ${ }^{8,9,13}$ As mulheres prevalecem em todas as faixas de idade, em especial entre os idosos com 80 anos e mais. A expectativa de vida aos 60 anos para as mulheres situa-se me torno de 19,3 anos, enquanto os homens idosos contam com um adicional de vida menor, de aproximadamente 16,8 anos. A razão de sexo corresponde a $120 \mathrm{mu}$ lheres para cada 100 homens, variando de 118 , para o grupo de 60 a 69 anos, até 141, nas idosas com 80 anos e mais. ${ }^{14,15}$

A escolaridade insuficiente ainda constitui um problema entre os mais velhos, principalmente entre as mulheres. Em 2000, os idosos que não sabiam ler ou escrever correspondiam a 35,2\% e, em 2002, a 33,2\%. No grupo sem escolaridade formal, as mulheres representavam $37,5 \%$ em 2000 e $35,3 \%$ em $2002.4,9$

De forma diferenciada ao observado para a população idosa em geral, na casuística estudada, $23,4 \%$ dos idosos têm escolaridade acima de 12 anos de estudo. Uma possível seletividade da clientela que procura por assistência à saúde no serviço ambulatorial analisado pode justificar em parte os achados divergentes aqui encontrados.

A escolaridade se configura como importante indicador de caracterização socioeconômica, relacionando-se às possibilidades de acesso à renda e utilização de serviços de saúde. ${ }^{13}$

O número maior de idosas viúvas em relação aos homens, aspecto também observado em outros estudos, respalda-se na maior dificuldade de recasamento das idosas após a viuvez, ao contrário do que ocorre em relação aos idosos. Normas sociais e culturais prevalecentes em nossa sociedade favorecem os homens, com a possibilidade de um novo casamento inclusive com mulheres mais jovens. Este fato, aliado à maior sobrevida feminina, contribui para a existência de um número significativo de mulheres sozinhas, principalmente nas faixas etárias mais avançadas. $2,4,6,9$

Em relação à pouca acessibilidade a um plano de saúde privado, verificada na grande maioria dos idosos que demandam ao ambulatório do NAI, apesar de a renda não ter sido incluída enquanto variável investigada, não se pode descartar a possibilidade de insuficiência financeira para fazer frente aos custos de manutenção do mesmo, sempre crescentes com o avançar dos anos. Registros na literatura especializada sinalizam a importância da renda do idoso, muitas vezes única fonte de custeio para toda a família. 14,16,17 $^{2}$

O tempo de permanência do idoso no serviço apresenta grande variabilidade, com 
média de 2 anos e 8 meses e desvio padrão de 1 ano e meio e coeficiente de variação da ordem de $50 \%$.

A construção do modelo teve por objetivo identificar quais variáveis, dentre as que compõem o cadastro do NAI, influenciam no tempo de permanência. O modelo evidencia que tanto para o nível 1 como para o nível 2 da variável dependente, a idade é significativa e contribui no sentido de reduzir a permanência. ${ }^{17} \mathrm{~A}$ existência de antecedentes cirúrgicos, assim como problemas de audição, urinários e fraturas ósseas, contribui para aumentar o tempo de permanência. Por outro lado, a existência de tonturas, problemas de visão e catarata contribui para reduzir sua permanência.

O efeito de mudança em uma unidade na variável independente é dado por: 0,91 ; 0,$499 ; 0,408 ; 0,2864 ; 3,296 ; 0,064 ; 12,007$; 3,691 , respectivamente, para as variáveis idade, tonturas, visão, audição, urinária, catarata, fraturas ósseas e antecedentes cirúrgicos. Em termos da razão de odds, isto representa que se o idoso tem problemas de tontura, problemas de visão, audição, problemas urinários, catarata, fraturas ósseas e antecedentes cirúrgicos, o mesmo tem metade da chance de permanecer no ambulatório por mais de um ano, em relação àquele que não apresenta este tipo de problema. Interpretação similar é dada para os outros efeitos analisados. Registra-se ainda que o coeficiente da variável idade é negativo, ou seja, à medida que a idade avança, diminui a expectativa de seu tempo de permanência no ambulatório do NAI.
Podem-se fazer predições individuais, através da expressão:

$P($ evento $)=\frac{1}{1+e^{-\left(\beta_{0}+\beta_{1} X_{1}+\beta_{2} X_{2}+\ldots+\beta_{n} X_{n}\right)}}$

onde os $b_{i}$ são os coeficientes e $\mathrm{X}_{\mathrm{i}}$ as variáveis independentes da equação de regressão (1).

Se o valor encontrado for menor que 0,05, o idoso é classificado como não tendo probabilidade de baixa permanência no NAI.

A identificação do perfil da clientela idosa demandada poderá contribuir de forma favorável no planejamento e implementação de atividades, em nível individual ou coletivo, mediante a realização de oficinas temáticas com vistas à integralidade da atenção à pessoa idosa, preconizada como um dos determinantes do envelhecimento ativo. Vale destacar que a Organização Mundial de Saúde considera envelhecimento ativo como o processo de otimização das oportunidades de saúde, participação e segurança, com o objetivo de melhorar a qualidade de vida à medida que as pessoas ficam mais velhas. ${ }^{18}$

\section{CONCLUSÃO}

Considerando a importância da organização e do planejamento de ações em serviços dirigidos à clientela idosa, segmento emergente que demanda cuidados específicos, os dados aqui investigados poderão subsidiar a estruturação de ações com vistas à integralidade da atenção em saúde no ambulatório geronto-geriátrico estudado. 


\section{REFERÊNCIAS}

1. Brasil. Decreto Lei, $n^{\circ} 8842$ de 04 de janeiro de 1994. Dispõe sobre a Política Nacional de Saúde do Idoso e cria o Conselho Nacional do Idoso. Brasília, DF: Ministério da Justiça, Secretaria Especial de Direitos Humanos;1998.

2. Chaimowicz F. Os idosos brasileiros no século XXI: demografia, saúde e sociedade. Belo Horizonte: Postgraduate; 1998.

3. Camarano AA, Pasinoto MT. O envelhecimento populacional na agenda das políticas públicas. In: Camarano AA, organizador. Os novos idosos brasileiros: muito além dos 60? Rio de Janeiro: IPEA; 2004. p.253-92.

4. Camarano AA. Envelhecimento da população brasileira: uma contribuição demográfica. Rio de Janeiro: IPEA; 2002.

5. Carmo EH, Barreto ML, Silva JB. Mudanças nos padrões de morbimortalidade da população brasileira: os desafios para um novo século. Epidemiologia e serviços de saúde: revista do Sistema Único de Saúde do Brasil 2003; 12 (2): 63-75.

6. Carvalho JAM, Garcia RA. O envelhecimento da população brasileira: um enfoque demográfico. Cad Saude Publica 2003; 19(3): 725 -33.

7. Silvestre JA; Kalache A; Ramos LR; Veras RP. O envelhecimento populacional brasileiro e o setor de saúde. Arquivos de geriatria e gerontologia 1996; 1(1): 81-9.

8. Veras RP. País jovem com cabelos brancos - a saúde do idoso no Brasil. Rio de Janeiro: Relume Dumará; 1994.

Recebido: 08/9/2008

Aprovado: 21/11/2008
9. Ramos LR. Determinant factors for healthy aging among senior citizens in large city: The Epidoso Project in São Paulo. Cad Saude Publica 2003; 19(3): 793 -8.

10. Soares JF; Colosimo EA. Métodos Quantitativos na pesquisa clínica. Ribeirão Preto: RBRAS; 1995.

11. Altman DG. Practical statistics for medical research. Londres: Chapman and Hall; 1991.

12. Kelsey JL, Thompson WD, Evans, AS, Whittemore AS. Methods in Observational Epidemiology. Oxford University Press; 1986. 366 p.

13. Araújo JC; Alves MIC. Perfil da população idosa no Brasil. Textos sobre Envelhecimento 2000;3 (3):7-19.

14. Beltrão KI, Camarano AA, Kanso, S. Dinâmica populacional brasileira na virada do século XX. Rio de Janeiro: IPEA; 2004.

15. Anderson MI. Saúde e condições de vida do idoso no Brasil. Textos sobre Envelhecimento 1998;1(1):7-22.

16. Telarolli Júnior $\mathrm{R}$, et al. Perfil demográfico e condições sanitárias dos idosos em área urbana do Sudeste do Brasil. Rev Saúde Pública 1996; 30(5):485-98.

17. Veras RP. A novidade da agenda social contemporânea: a inclusão do cidadão de mais idade. A Terceira Idade 2003; 14(28): 6-29.

18. Vieira S. Bioestatística: tópicos avançados, 2. ed. Rio de Janeiro: Campus Elsevier Science; 2003.

19. Organização Mundial de Saúde. Envelhecimento ativo: uma política de saúde. Brasília: Organização PanAmericana de Saúde; 2005. 